Mayra Matesanz ${ }^{1}$

Ana Poza ${ }^{1}$

Amaia Iñurrieta ${ }^{1}$

Eddita Fernández-Diaz'

Marta Arroyo ${ }^{1}$

Ignacio Dominguez ${ }^{2}$

Francisco Javier Cande ${ }^{3}$

\title{
Dalbavancin was effective and safe after one year of treatment in a complicated osteoarticular infection caused by methicillin-resistant Staphylococcus aureus
}

\author{
${ }^{1}$ Hospital at Home Unit. Hospital Clínico San Carlos. Madrid. Spain \\ ${ }^{2}$ Spine Unit. Traumatology and Orthopaedic Surgery. Hospital Clínico San Carlos. Madrid. Spain \\ ${ }^{3}$ Clinical Microbiology and Infectious Diseases. IdISSC and IML Health Institutes Hospital Clínico San Carlos. Madrid. Spain
}

Article history

Received: 1 February 2021; Revision Requested: 25 March 2021; Revision Received: 27 March 2021; Accepted: 1 April 2021; Published: 6 May 2021

\section{Sir,}

Osteomyelitis is a subacute/chronic infectious process that is often perpetuated by the presence of contaminated osteosynthesis material, which cannot always be removed due to the surgical risk involved. The success rate in chronic infection after removal and with prolonged antimicrobial treatment is less than $40 \%$, leading to suppressive antibiotic strategies which in more than 50\% of cases are withdrawn due to toxicity. Dalbavancin is a long-acting lipopeptide that has demonstrated outstanding in vitro activity against resistant Gram-positive bacteria. There is evidence for the effectiveness and safety of dalbavancin in biweekly dosing schedules. This could be useful in the treatment of infections that require prolonged treatment in home care units. However, we were unaware of the presence of side effects in suppressive treatments. We present the case of a young patient with infantile cerebral palsy, with functional dependence and a coxofemoral osteoarticular infection complicated by methicillin-resistant Staphylococcus aureus (MRSA), perpetuated by the migration of a Harrington bar used for vertebral fixation that invaded the joint. Intravenous dalbavancin was administered at a dose of $1,500 \mathrm{mg}$ every 15 days for one year. Clinical and analytical parameters were determined to assess efficacy and safety.

A 41-year-old male with a history of chronic ischaemic-anoxic motor ischaemic encephalopathy of perinatal origin with total body involvement and tetraparietal expansion, symptomatic epilepsy since the age of 13-14 years, related to the encephalopathy. He has had a gastrostomy tube for more than 10 years due to swallowing spasticity with propulsive defect and aspiration pneumonia. Severe neuropathic scoliosis and operated in 1996 with Harrington bars and Luque wires.

Correspondence:

Francisco Javier Candel

Clinical Microbiology and Infectious Diseases

IdISSC and IML Health institutes

Hospital Clinico San Carlos. Madrid. Spain.

E-mail: franciscojavier.candel@salud.madrid.org
Under treatment with lacosamide, phenobarbital, prokinetics, laxatives for constitutional constipation and protein supplements.

From July 2018 to March 2019 the patient developed up to four episodes of fever, lumbar pain, right coxalgia, wound on the external and upper thigh and a palpable collection (figure 1). In all of them, blood tests revealed leukocytosis with left shift, as well as elevated CRP levels. The CT scan showed Harrington bar migration, coxofemoral joint invasion and contiguous collection (figure 2). In the culture of the collection, MRSA was isolated in all episodes in association with Proteus mirabilis with different antibiogram phenotypes (figure 1, table 1). On up to three occasions the case was brought to the attention of the Spine Unit of the Traumatology and Orthopaedic Surgery Department, which rejected the removal of osteosynthesis material due to the high surgical risk involved. Suppressive treatment was started for 6 weeks, keeping the patient afebrile and with a progressive decrease in biomarkers (CRP). The suppressive treatment regimens were initially combinations of linezolid $600 \mathrm{mg}$ every 12 hours associated with ceftriaxone $2 g$ every 24 hours iv, In the first cycle of treatment the patient developed thrombopenia and cholestasis. Subsequent treatment regimens were performed with daptomycin $10 \mathrm{mg} / \mathrm{kg} /$ day and ertapenem $1 \mathrm{~g} / 24 \mathrm{~h}$. After the third episode of clinical recurrence, the case was discussed again with the Spine Unit who accepted the surgical revision. The Harrington Bar that caused the invasion of the coxofemoral joint was removed. The contralateral one and the Luque wires, which were included in the spinal arthrodesis, were maintained.

Two months after removal, the patient returned to the emergency department with fever $38.5^{\circ} \mathrm{C}$, a spontaneous fistula with abundant purulent content, leukocytosis with left deviation and a CRP of $18.6 \mathrm{mg} / \mathrm{dl}$. In the culture of the purulent material, MRSA with the same phenotype was reisolated. Suppressive treatment with dalbavancin $1500 \mathrm{mg}$ every 15 days was started, with early clinical improvement, and the patient remained afebrile, and CRP decreased. During the months of 


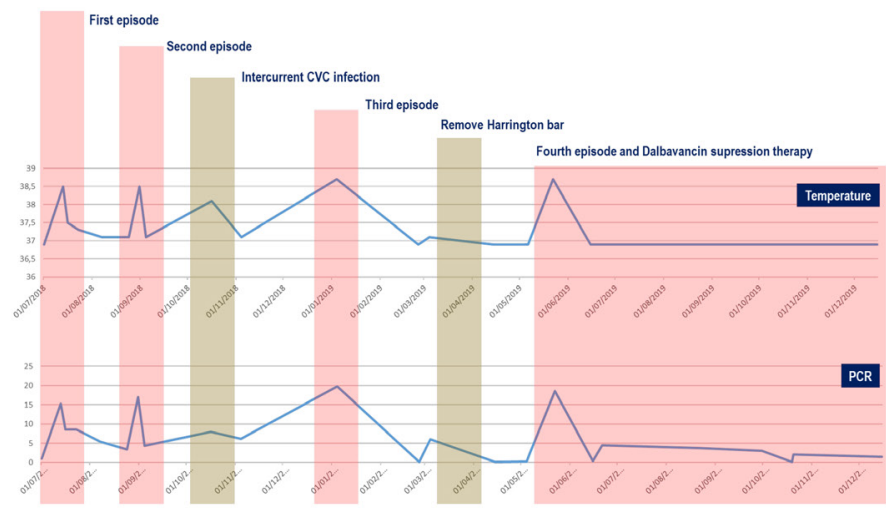

A

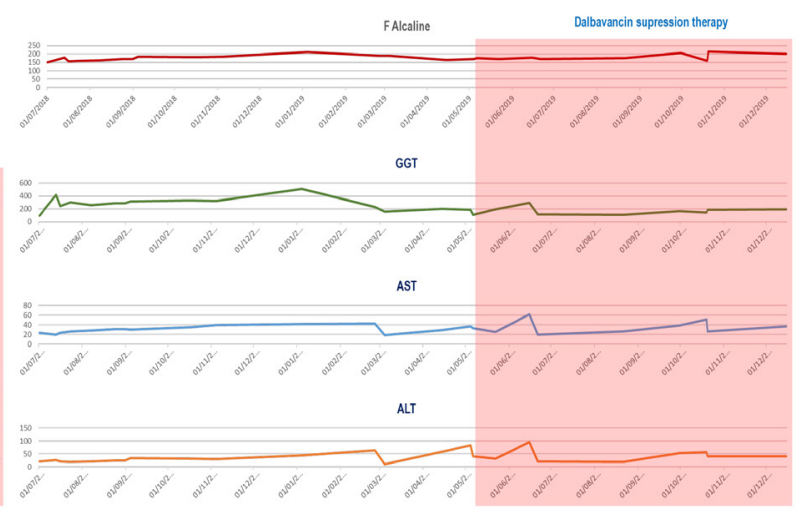

B

Figure 1 A: Temperature and PCR monitization during process. B: Monitorizaction of hepatic profile during process.
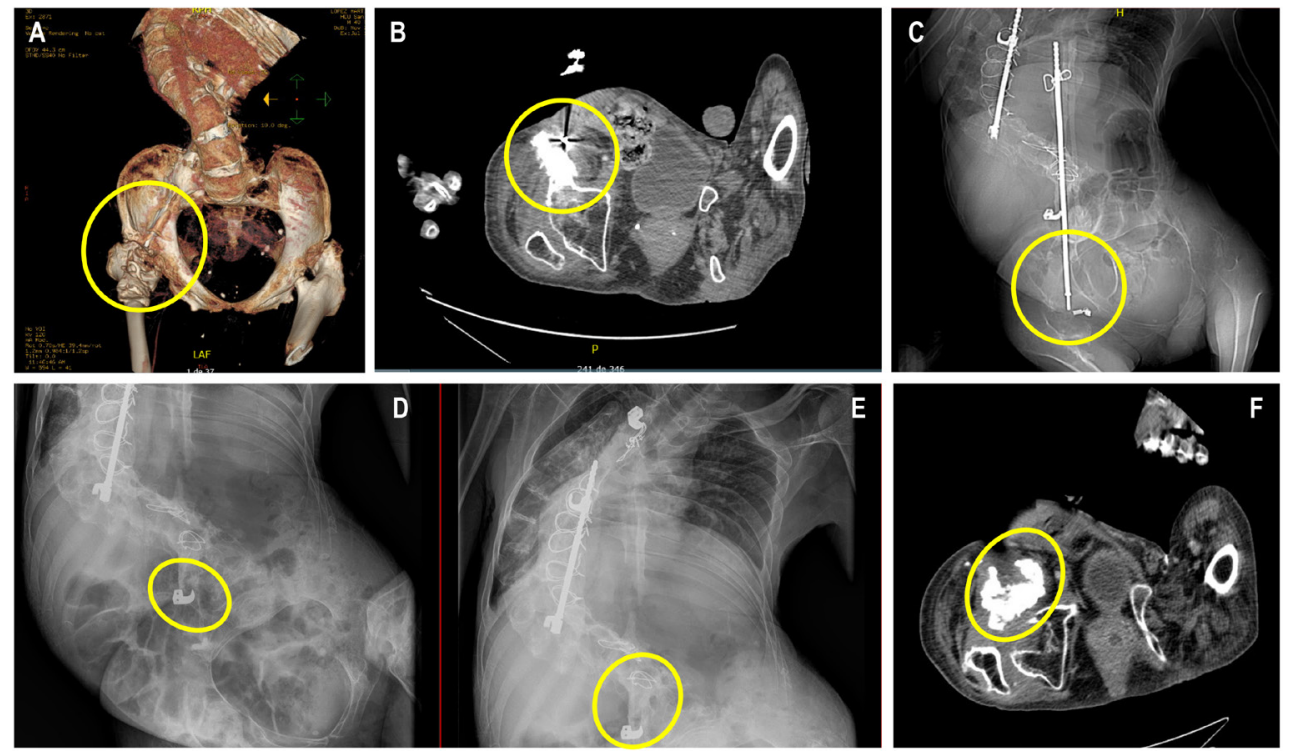

Figure $2 \quad A, B$ and C (13/7/2018). Migrated Harrington bar invading coxofemoral joint. $D$ and $E(4 / 3 / 2019)$. Removal of the migrated Harrington bar, paravertebral approach. Impossibility to remove wire ties. F: (27/1/2020). Almost complete dislocation and destruction of the right coxofemoral joint. Zones of bone proliferation and areas of low attenuation suggestive of intra-articular collections in resolution are identified.

the COVID-19 pandemic, she continued to be monitored by telephone and analytical controls did not develop any clinical signs of toxicity or liver biochemical alterations (figure 1). The radiological control of January 2020 by CT reported a dislocation and almost complete destruction of the right coxofemoral joint. Areas of bony proliferation and areas of low attenuation suggestive of intra-articular collections were identified in resolution (figure 2F). Treatment was maintained every 15 days until 29 June 2020, with weekly, fortnightly, and then monthly monitoring of temperature, CRP and liver function parameters. At review in December 2020 and January 2021, he remained asymptomatic.

Dalbavancin is a long-acting lipopeptide with a fatty acid chain responsible for its long-estimated half-life of up to 14 days, which has demonstrated extraordinary in vitro activity against resistant Gram-positive strains, specifically MRSA, where it has a $\mathrm{MIC}_{50}$ of $0.03 \mathrm{mg} / \mathrm{l}$ and a $\mathrm{MIC}_{90}$ of $0.12 \mathrm{mg} / \mathrm{l}$, being 10 times more potent than vancomycin and 10 times more 


\section{Table $1 \quad$ Staphylococcus aureus isolation with the same phenotype in all cultures (July and August 2018, January 2019). Proteus mirabilis isolation with different phenotypes.}

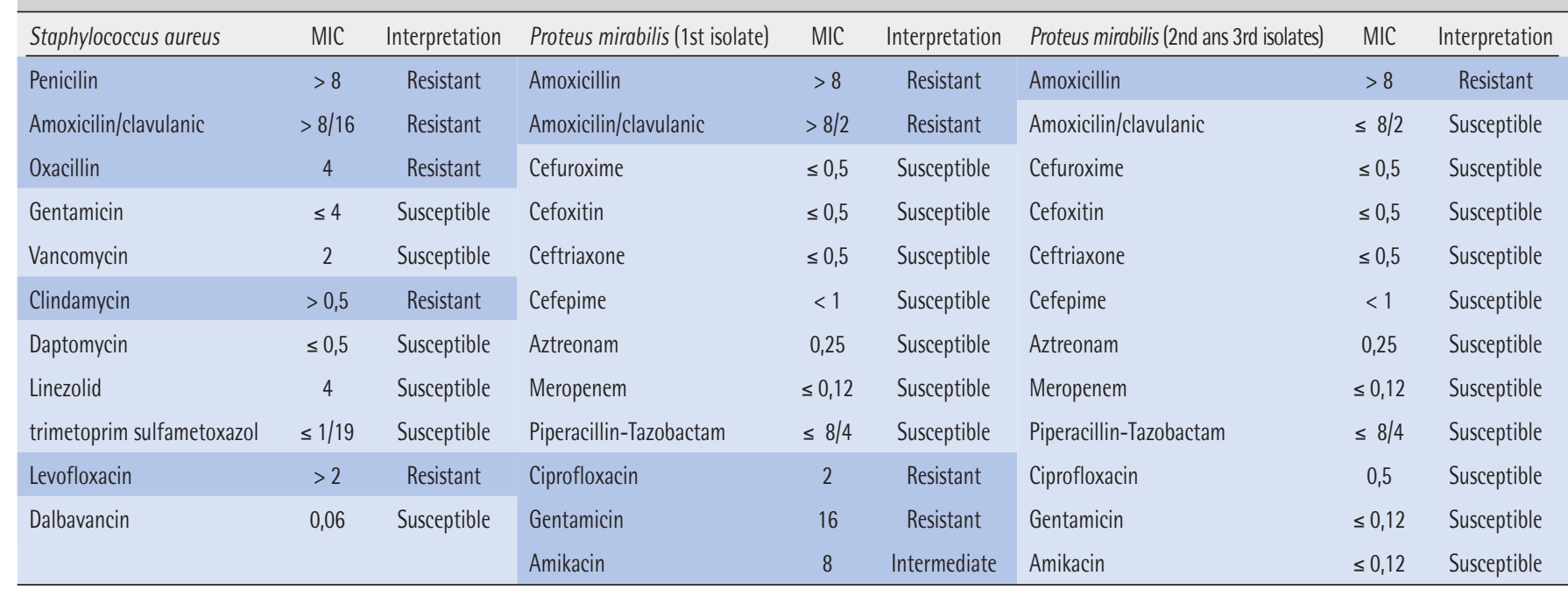

potent than linezolid $[1,2]$. It has a protein binding of 93\%, its elimination half-life is 372 hours (333-405) and it maintains a concentration of more than $8 \mathrm{mg} / \mathrm{L}$ for one month, both after infusion at a dose of $1000 \mathrm{mg}$ on the first day, adding $500 \mathrm{mg}$ a week, and maintaining a dose of $1500 \mathrm{mg}$ every two weeks [3].

In clinical studies in skin and soft tissue infection, no differences were found with respect to sequential therapy with vancomycin and linezolid [4]. In the comparative study on skin and soft tissue infection, the primary efficacy and safety endpoints between different dalbavancin strategies. No differences were found at 14 days in the $20 \%$ reduction in erythema 48-72 hours after initiation of therapy, in clinical success or in adverse effects between the fractionated treatment schedule of 1,000 mg first dose with a second $500 \mathrm{mg}$ per week or a single dose schedule of 1,500 mg [5], with both doses included in its data sheet. Dalbavancin is also active in the bacterial population embedded in the biofilm. Experimental models suggest that dalbavancin is distributed in bone, skin and joint tissue at concentrations above the MIC of S. aureus over long periods of time even after a significantly reduced dosing regimen [6], which would suggest prolonged treatment strategies with lower than standard doses while maintaining effectiveness [7].

In clinical studies in skin and soft tissue infection, there is no evidence that dalbavancin is distributed in bone, skin and joint tissue at concentrations expected to exceed the MIC for $\mathrm{S}$. aureus over long periods of time after a significantly reduced dosing regimen [7], which would even suggest prolonged treatment strategies with lower than standard doses while maintaining effectiveness. There is also evidence of little or no toxicity during prolonged treatment in patients who have had toxicity with other drugs previously and in whom osteosynthesis material cannot be removed $[8,9]$.
Suppressive therapy was maintained for 1 year after removal of part of the osteosynthesis material with excellent tolerance and the patient remained asymptomatic 6 months after the end of treatment. The long half-life, which even allows removal of permanent vascular access in prolonged treatment, the in vitro activity against all Gram-positive bacteria, the activity in biofilm and its excellent tolerability make this antibiotic an extraordinarily useful tool in home hospitalisation units in general and particularly in suppressive strategies in comorbid patients when the implant cannot be removed.

\section{FUNDING}

None to declare

\section{CONFLICTS OF INTEREST}

The authors declare that they have no conflict of interest.

\section{REFERENCES}

1. Biedenbach DJ, Bell JM, Sader HS, Turnidge JD, Jones RN. Activities of dalbavancin against a worldwide collection of 81,673 gram-positive bacterial isolates. Antimicrob Agents Chemother. 2009;53(3):1260-3. doi: 10.1128/AAC.01453-08.

2. Jones RN, Sader HS, Flamm RK. Update of dalbavancin spectrum and potency in the USA: report from the SENTRY Antimicrobial Surveillance Program (2011). Diagn Microbiol Infect Dis. 2013;75(3):304-7. doi: 10.1016/j.diagmicrobio.2012.11.024.

3. Dorr MB, Jabes D, Cavaleri M, Dowell J, Mosconi G, Malabarba A, White RJ, Henkel TJ. Human pharmacokinetics and rationale for once-weekly dosing of dalbavancin, a semi-synthetic glycopeptide. 
J Antimicrob Chemother. 2005;55 Suppl 2: ii25-30. doi: 10.1093/ jac/dki008.

4. Boucher HW, Wilcox M, Talbot GH, Puttagunta S, Das AF, Dunne MW. Once-weekly dalbavancin versus daily conventional therapy for skin infection. N Engl J Med. 2014;370(23):2169-79. doi: 10.1056/NEJMoa1310480.

5. Dunne MW, Puttagunta S, Giordano P, Krievins D, Zelasky M, Baldassarre J. A Randomized Clinical Trial of Single-Dose Versus Weekly Dalbavancin for Treatment of Acute Bacterial Skin and Skin Structure Infection. Clin Infect Dis. 2016;62(5):545-51. doi: 10.1093/cid/civ982. PMID: 26611777;

6. Knafl D, Tobudic S, Cheng SC, Bellamy DR, Thalhammer F. Dalbavancin reduces biofilms of methicillin-resistant Staphylococcus aureus (MRSA) and methicillin-resistant Staphylococcus epidermidis (MRSE). Eur J Clin Microbiol Infect Dis. 2017; 36(4): 677. PMID: 27896496

7. Dunne MW, Puttagunta S, Sprenger CR, Rubino C, Van Wart $S$, Baldassarrea J. Extended-Duration Dosing and Distribution of Dalbavancin into Bone and Articular Tissue. Antimicrob Agents Chemother. 2015; 59(4): 1849-1855. doi: 10.1128/AAC.04550-14. PMID: 25561338

8. Morata $L$, Cobo J, Fernández-Sampedro $M$, Guisado $P$, Ruano $E_{1}$ Lora-Tamayo J, et al. Safety and Efficacy of Prolonged Use of Dalbavancin in Bone and Joint Infections. Antimicrob Agents Chemother. 2019; 63(5): e02280-18. doi: 10.1128/AAC.02280-18. PMID: 30858217

9. Buzón L, Mora-Fernández M, Perales JM, Ortega M, Álvarez-Paredes L, Morán MA,et al. Dalbavancin for treating prosthetic joint infections caused by Gram-positive bacteria: A proposal for a low dose strategy. A retrospective cohort study" Rev Esp Quimioter. 2019; 32(6): 532-538. PMID: 31642637. 